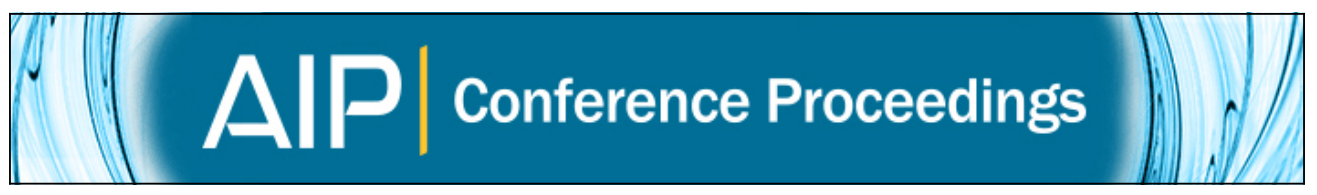

\title{
Photometric Variability and Rotation in Magnetic White Dwarfs
}

K. A. Lawrie, M. R. Burleigh, C. S. Brinkworth, and T. R. Marsh

Citation: AIP Conference Proceedings 1273, 91 (2010); doi: 10.1063/1.3527897

View online: http://dx.doi.org/10.1063/1.3527897

View Table of Contents:

http://scitation.aip.org/content/aip/proceeding/aipcp/1273?ver=pdfcov

Published by the AIP Publishing

Articles you may be interested in

White Dwarf Pulsations in the Cataclysmic Variables SDSS J0919+08 and VSX J0747+0650 AIP Conf. Proc. 1273, 526 (2010); 10.1063/1.3527880

Ultracool Companions to White Dwarfs

AIP Conf. Proc. 1273, 384 (2010); 10.1063/1.3527847

White Dwarfs in NGC6397 and M4: Constraints on the Physics of Crystallization

AIP Conf. Proc. 1273, 146 (2010); 10.1063/1.3527792

The White Dwarf Age of NGC 2477

AIP Conf. Proc. 1273, 135 (2010); 10.1063/1.3527789

White Dwarf and Pre-White Dwarf Pulsations

AIP Conf. Proc. 1170, 605 (2009); 10.1063/1.3246571 


\title{
Photometric Variability and Rotation in Magnetic White Dwarfs
}

\author{
K. A. Lawrie*, M. R. Burleigh*, C. S. Brinkworth ${ }^{\dagger}$ and T. R. Marsh** \\ ${ }^{*}$ Dept. of Physics and Astronomy, University of Leicester, University Rd., Leicester, LE1 7RH, UK \\ ${ }^{\dagger}$ Spitzer Science Center, California Institute of Technology, Pasadena, CA 91125, USA \\ ${ }^{* *}$ Dept. of Physics, University of Warwick, Coventry, CV4 7AL, UK
}

\begin{abstract}
We present a search for long term (months - years) photometric variability in a sample of ten isolated magnetic white dwarfs using observations taken with the Liverpool Robotic Telescope between March 2005 and January 2007. These stars had previously been found to be photometrically stable on short (hours - one week) timescales [1]. We construct differential light curves for each target and then use CLEAN and Lomb-Scargle periodograms to determine any periodicity that may be present. Photometric variability is detected in two of the targets during the observed timescale - G 240-72 and G 227-28. We find no variability in the remaining eight targets above the $1 \%$ level. Finally, we search for any correlations between the spin periods and intrinsic physical properties of magnetic white dwarfs, such as the magnetic field strength, temperature, mass and age.
\end{abstract}

Keywords: stars:white dwarfs - magnetic fields - rotation

PACS: 97.20.Rp, 97.10.Kc, 97.10.Ld

\section{INTRODUCTION}

Magnetic fields $(10 \mathrm{kG}<B<1000 \mathrm{MG})$ have been measured in approximately 200 isolated white dwarfs (e.g. [2,3]). The origin of magnetic fields in white dwarfs still remains largely uncertain. The chemically peculiar magnetic main-sequence Ap and Bp stars have been proposed as the progenitors of high-field magnetic white dwarfs (MWDs) ( $1 \mathrm{MG}<B<1000 \mathrm{MG}$ ) [4]. On the other hand, [5] suggested that MWDs have a binary origin and are the result of merger at the end of a common envelope evolution.

Rotation rates of non-magnetic white dwarfs are very difficult to measure as their spectral lines undergo considerable broadening due to the high surface gravity. However, rotation periods can be measured for MWDs using variable photometric or circular polarization observations. It is estimated that $\sim 50 \%$ of MWDs show photometric periodic variability as the star rotates $[1,6]$. Therefore, isolated MWDs are particularly important for understanding the evolution of the general population of white dwarfs.

Photometric variations in high-field MWDs are due to the effects of magnetic dichroism, the dependence of the continuum opacity on the surface field strength [7]. However, photometric variability has also been observed in cool, low-field MWDs $(T<12000 \mathrm{~K})$, where the modulations have been attributed to star spots on the surface of the white dwarf in a convective atmosphere $[8,9]$. The reduced convection at the locations of the spots means they are cooler (and therefore darker), leading to a drop in the observed brightness of the star as the spots rotate into view. This effect was observed in WD 1953-011 $\left(T_{\text {eff }} \approx 8000 \mathrm{~K}, B \approx 70 \mathrm{kG}\right)$ which displayed $\approx 2 \%$ peak-to-peak amplitude sinusoidal variability every 1.44 days due to a star spot covering $\sim 10 \%$ of the surface [10, 9]. 
Precise measurements of rotation periods of a large sample of white dwarfs are required to test theories of mass and angular momentum loss during the post mainsequence evolution, and the formation and evolutionary scenarios to explain the generally lower than expected rotation periods of white dwarfs.

\section{OBSERVATIONS AND DATA ANALYSIS}

Observations of 13 targets were carried out using the Liverpool Robotic Telescope (LT) with the RATCam Instrument in the SDSS $r^{\prime}$ filter on La Palma between March 2005 and January 2007. These targets were selected from a previous survey by [1, 6], which were found to be photometrically stable on short timescales (hours - one week) but showed long term modulations between observing seasons. Three targets in the sample were not analyzed due to poor data quality or an insufficient number of epochs. Differential photometry was performed on each target with respect to bright comparison stars in the field. For each target, we used a CLEAN periodogram [11] and Lomb-Scargle periodogram $[12,13]$ within the Starlink Package PERIOD to search for periodicity in the differential light curves. The errors quoted with the periods were calculated by fitting a Gaussian curve to the peak of interest and finding the FWHM.

\section{RESULTS}

Variability was detected in two out of the ten targets analyzed (G 240-72 and G 227-28), and no modulations above the $\pm 1 \%$ level were found in the remaining targets.

G 240-72: The polarization of G 240-72 was reported by [14] to vary extremely slowly, suggesting it was a very slow rotator with a period of $\geq 100$ years. No evidence for short term variability was found by [6], however they do report an increase in flux of $\sim 2.5 \%$ over 10 months. We detect variability of $\sim 4.5 \%$ peak-to-peak amplitude in our differential light curve over 20 months (Fig. 1), while the differential light curve of the comparison stars shows variability of $\sim 1.5 \%$ peak-to-peak (Fig. 1), demonstrating that G 240-72 varies photometrically.

Peaks were identified at $56.3 \pm 2$ days $(0.01776$ cycles/day $)$ and $16.3 \pm 0.2$ days ( 0.06135 cycles/day) in the CLEAN and Scargle periodograms (Fig. 2) and neither of the peaks were detected in the analysis of the comparison stars. In addition, we find no evidence of a peak around 29.53 days (lunar cycle), meaning the 56 day period is not likely associated with the lunar cycle. The 16 day period is dubious as [6] would have likely found evidence for such a periodicity over an intensive week of observations. In addition, the 16 day period could be associated with the mean sampling rate at 32.6 days. The light curves were folded on both periods and a sine curve was fitted. The reduced $\chi^{2}$ statistic was comparable in both cases for the 56 day and 16 day period, with 10.38 and 10.67 respectively, illustrating that neither was a particularly good fit. A constant fit to the light curve gave a reduced $\chi^{2}$ of 17.50 , which is much larger than the statistic from the sine curve fit, thus further suggesting that the target is variable. Consequently, we have been unable to determine a reliable period of variability for G 240-72, although we believe it to be varying photometrically on the timescale of months to years. 

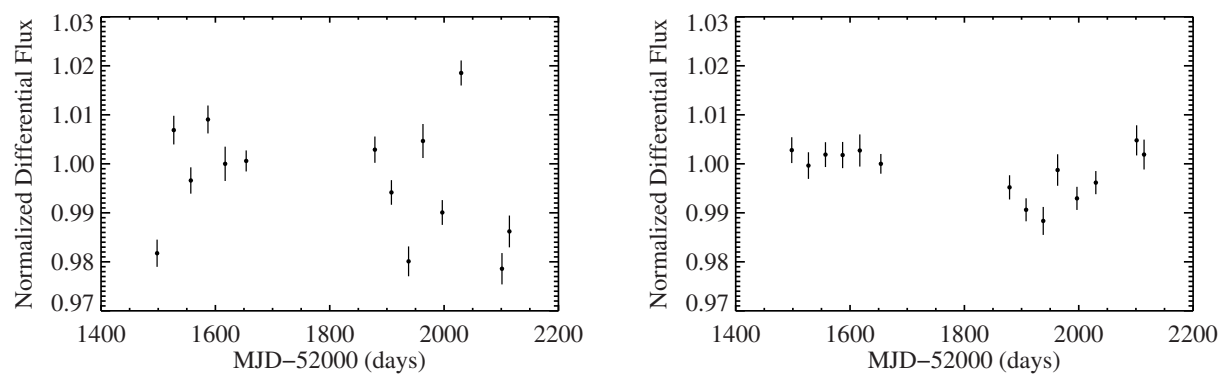

FIGURE 1. Differential light curves of G 240-72. Left panel: Target differential light curve. Right panel: Differential light curve of the comparison stars.
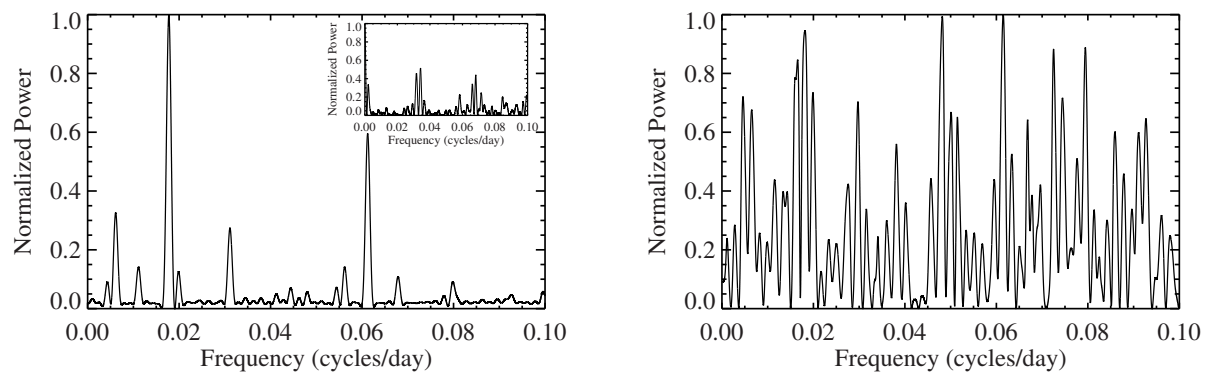

FIGURE 2. Left panel: CLEAN periodogram for G 240-72. Two peaks are detected in the CLEAN periodogram at $56.3 \pm 2$ days ( 0.01776 cycles/day) and $16.3 \pm 0.2$ days $(0.06135$ cycles/day). Left panel inset: Fourier transform of the window function. Right panel: Scargle periodogram for G 240-72. The two peaks are also seen in the Scargle periodogram, although there are many other features.

G 227-28: No evidence was found for short term variability in G227-28 by [6], but they did report a $2 \%$ difference in flux over several months and concluded it was variable on timescales of months to years. We find variability of $\sim 3 \%$ in our differential light curve over 17 months (Fig. 3), although the scatter in the differential flux of the comparison stars is substantial at $\sim 2 \%$ (Fig. 3). We find a best-fitting period of $16.0 \pm 0.3$ days from the Lomb-Scargle periodogram, but we also detect another strong peak at $67 \pm 7$ days. Despite the 16 day period providing the best-fit, we feel it is an unlikely result as it would have persumably been at least partially detected by [6] during a week of observations, but the period could also be related to the mean sampling rate at 33.15 days. When the light curve was folded on the 16 day period the reduced $\chi^{2}$ of the fitting sine curve was 2.26. Similarly, fitting a sine curve to the 67 day period gave a reduced $\chi^{2}$ of 3.01. A constant fit to the differential light curve gave a reduced $\chi^{2}$ of 6.39. We conclude that we find photometric variability in G227-28 (similar to previous findings from [6]), although we are unable to determine a period of variability.

Grw+70 ${ }^{\circ}$ 8247: This MWD is also thought to be a very slow rotator with a period 

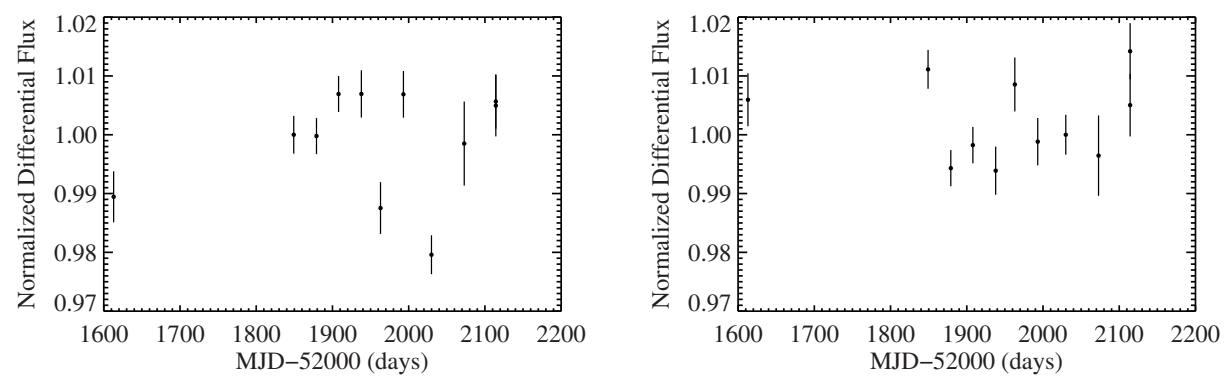

FIGURE 3. Differential light curves of G 227-28. Left panel: Target differential light curve. Right panel: Differential light curve of the comparison stars.

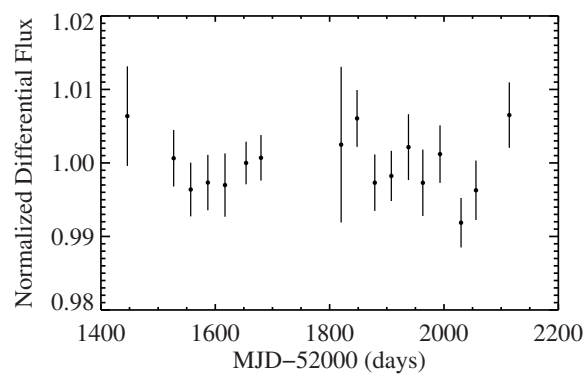

FIGURE 4. Grw $+70^{\circ} 8247$ differential light curve over 22 months of observations between March 2005 and January 2007.

$\geq 100$ years [14]. We find no variability in Grw $+70^{\circ} 8247$ over the 22 month period of observations. A constant fit to the differential light curve (Fig. 4) gives a reduced $\chi^{2}$ of 0.94 . This result agrees with the hypothesis that it is a very slow rotator.

We searched for correlations between the well-defined rotation rates of MWDs and other intrinsic physical parameters, such as the magnetic field strength, temperature, mass, and age (Fig. 5) [1, 6]. We may have detected a weak, negative correlation between rotation period and magnetic field strength (also found by [1]), but there also seems to be an interesting trend emerging between the spin period and age. The small number of data points could imply a spin down effect, although many more well-constrained rotation periods will be required to determine whether or not it is a genuine correlation.

\section{DISCUSSION}

The photometric variability detected in G 240-72 and G 227-28 is consistent with previous findings from $[1,6]$, although unfortunately we have been unable to determine a reliable period of variability in either case. It appears that the de-rotator on the LT was 

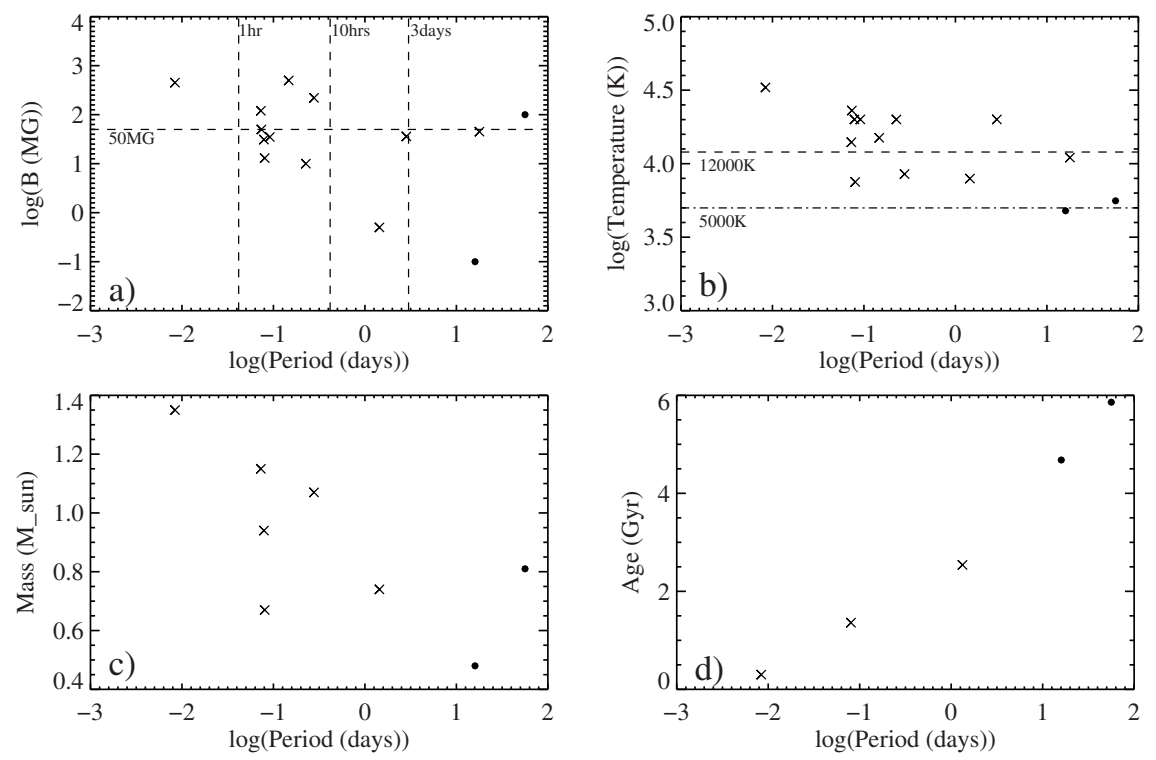

FIGURE 5. Rotation period versus intrinsic physical properties of magnetic white dwarfs - a) magnetic field strength, b) temperature, c) mass, and d) age. The crosses include targets from [1, 2]. Information on the physical parameters was obtained from $[1,6,15,16,17]$. The filled circles are the best fit periods for $\mathrm{G} 240-72$ ( $\mathrm{P}=56$ days) and $\mathrm{G} 227-28$ ( $\mathrm{P}=16$ days).

not operational for some of the observations, and as a result the star fields around the target could differ vastly between epochs. Therefore, it was challenging to make a consistent comparison star selection for all of the epochs. This also meant that the target and comparison stars frequently fell on different parts of the CCD, introducing possible systematic uncertainties. In addition, the comparison stars often exhibited more scatter than the target, and consequently obtaining a reliable differential flux was difficult. We note that the photometric errors (which have been used here in the light curves) underestimate the true uncertainty in differential flux for each epoch. We also note that the spectral types of the comparison stars have not been checked, and therefore any substantial changes in the airmass between epochs could cause fluctuations in the differential flux that have not been accounted for. We notice trailed sources in the 2005 data in particular, which could compromise the photometric accuracy of the data. Furthermore, many of the observations were taken on a monthly basis rather than being randomly scheduled, making it difficult to search for a range of periodicities over the long timescale. These factors meant reliable and accurate detections could not be made below the $\pm 1 \%$ level.

We are currently expanding our sample size to include MWDs discovered by the Sloan Digital Sky Survey (SDSS) [3, 16]. To date, we have observed 27 SDSS MWDs using the INT on La Palma in March 2009, October 2009, and March 2010 and have also continued to follow-up suspected variable MWDs found by [1, 6]. This should dramatically increase the number of well-defined rotation periods measured for MWDs 
and therefore hopefully put constraints on the correlations shown in Fig 5.

\section{CONCLUSIONS}

Modulations on the timescale of weeks to months were detected in two out of the ten bright MWDs analyzed, which had previously been found to be photometrically stable on short timescales (hours to one week). Photometric variability of $\sim 4.5 \%$ peak-to-peak is found for G 240-72, while G 227-28 shows modulations of 3\% although there is considerable scatter in the differential flux of the comparison stars. Unfortunately, we have not been able to determine a reliable rotation period in either case. We may have found a weak negative correlation between the spin period and magnetic field strength of MWDs (see Fig 5, also reported by [1]). A relationship may also be emerging between rotation periods and age, suggesting a spin down effect. However, many more welldefined rotation periods are required to constrain any correlations between the rotation periods and intrinsic physical parameters of magnetic white dwarfs.

\section{ACKNOWLEDGMENTS}

The Liverpool Telescope is operated on the island of La Palma by Liverpool John Moores University in the Spanish Observatorio del Roque de los Muchachos of the Instituto de Astrofisica de Canarias with financial support from the UK Science and Technology Facilities Council. The authors acknowledge the data analysis facilities provided by the Starlink Project which is run by CCLRC on behalf of PPARC. This research has made use of the SIMBAD database, operated at CDS, Strasbourg, France.

\section{REFERENCES}

1. C. S. Brinkworth, M. R. Burleigh, and T. R. Marsh, "A Survey for Photometric Variability in Isolated Magnetic White Dwarfs - Measuring their Spin Periods," in 15th European Workshop on White Dwarfs, edited by R. Napiwotzki, and M. R. Burleigh, 2007, vol. 372, p. 183.

2. D. T. Wickramasinghe, and L. Ferrario, PASP 112, 873-924 (2000).

3. B. Külebi, et al., $A \& A \mathbf{5 0 6}, 1341$ (2009).

4. D. T. Wickramasinghe, and L. Ferrario, MNRAS 356, 1576 (2005).

5. C. A. Tout, et al., MNRAS 387, 897 (2008).

6. C. S. Brinkworth, M. R. Burleigh, and T. R. Marsh, (in preparation).

7. L. Ferrario, et al., MNRAS 292, 205 (1997).

8. C. S. Brinkworth, et al., MNRAS 348, L33 (2004).

9. C. S. Brinkworth, et al., MNRAS 357, 333 (2005).

10. P. F. L. Maxted, et al., MNRAS 315, L41 (2000).

11. D. H. Roberts, J. Lehár, and J. W. Dreher, AJ 93, 968 (1987).

12. N. R. Lomb, Ap\&SS 39, 447 (1976).

13. J. D. Scargle, ApJ 263, 835 (1982).

14. J. R. P. Angel, E. F. Borra, and J. D. Landstreet, ApJS 45, 457 (1981).

15. P. Bergeron, S. K. Leggett, and M. T. Ruiz, ApJS 133, 413 (2001).

16. A. Kawka, et al., ApJ 654, 499 (2007).

17. B. Külebi, et al., arXiv:1007.4978(2010). 